\title{
Early Toxicity Screening and Selection of Lead Compounds for Parasitic Diseases
}

\author{
Renata Campos Nogueira ${ }^{1}$, José Fernando Oliveira-Costa ${ }^{1}$, Matheus Santos de Sá1, Ricardo Ribeiro \\ dos Santos ${ }^{1,2}$ and Milena Botelho Pereira Soares ${ }^{1,2, *}$
}

${ }^{1}$ Centro de Pesquisas Gonçalo Moniz, Fundação Oswaldo Cruz. Rua Waldemar Falcão, 121. Candeal 40296-710 - Sal-
vador, BA - Brazil and ${ }^{2}$ Hospital São Rafael. Av. São Rafael, 2152. São Marcos 41253-190 - Salvador, BA, Brazil

\begin{abstract}
Despite many advances made in disease mechanisms knowledge and drug discovery and development processes, the election of promising lead compounds continues to be a challenge. Efficient techniques are required for lead selection of hit compounds selected through in vitro pharmacological studies, in order to generate precise low cost throughput data with minimal amount of compound to support the right decision making. In this context, the selection of lead compounds with physicochemical parameters that will benefit orally bioavailable drugs are crucial for patients compliance and cost effectiveness, as well as for successful pharmacology. A concept based in Lipinski's rules point out the importance of analyzing these informations in early stages. A hepatocyte screening system may provide data on many processes such as drug-drug interaction, metabolite formation, drug toxicity and ADME profile of a hit. Drug-induced liver injury is the most frequent reason for the withdrawal of an approved drug from the market and hepatocytes have a central role in the metabolism of xenobiotics. Cytotoxicity screening assays can also give some information about toxicity early drug discovery process. A set of goals in lead compound selection must be shared between all areas involved so the chances of success can be improved in translational research.
\end{abstract}

Key Words: Toxicity screening, lead compound, parasitic diseases, drug discovery.

\section{INTRODUCTION}

The search for new and improved treatments for diseases endemic of developing countries is increasing. Bacterial, protozoan and helminth infectious diseases such as tuberculosis, malaria, African sleeping sickness, leishmaniasis, Chagas' disease, onchocerciasis, lymphatic filariasis and schistosomiasis are among them. Although there is a need for new drugs to reduce mortality and morbidity caused by the mentioned infections [1], high cost, poor compliance, drug resistance, low efficacy and poor safety are limiting factors for drug development in resource-poor settings where these diseases occur.

Public-private partnerships have proved that they can move compounds quickly through the $R \& D$ process [2]. The Medicines for Malaria Venture is an example of publicprivate partnerships working on diseases for the developing world [3]. Gaps between basic research and clinical development can be transposed by joining expertise from academia, public sector and pharmaceutical industry.

R\&D costs increase substantially as compounds move through each successive phase (Fig. (1)). Directing the focus of $R \& D$ program to a fewer lead candidates would represent costs reduction and maximization of efforts towards a more promising drug candidate [4]. In this context, basic research

\footnotetext{
*Address correspondence to this author at the Milena Botelho Pereira Soares, Rua Waldemar Falcão, 121 - Candeal, Salvador, BA. 40296-710. Brazil; Tel: +55 71 3176-2260; Fax: +55 71 3176-2272;

E-mail: milena@bahia.fiocruz.br
}

(in vitro and in vivo pharmacology and toxicology assays) needs to share the same goals of pharmaceutical industry in order to supply leads for translational research.

A hit compound identified by the in vitro activity screening of drug design, natural products or chemical libraries has to be efficacious in disease animal models with no evident toxicity at efficacious doses in order to be called a lead compound. Effective networks help to establish a library that can be constructed based on pharmacologic, toxicological and pharmacokinetic properties of a lead compound in order to draw a drug candidate, which is an optimized lead compound that can be compared to drug standard activity with acceptable pharmacokinetic and toxicity profile [1].

Nowadays a hit is selected by biological activity and later on the properties related to its "drugability", i.e., ADME/ toxicity, are investigated [5]. Cytotoxicity should also be evaluated in the beginning of a drug screening program, in order to identify among the highly active compounds those excessively cytotoxic and thus incapable to be classified as lead compounds [6].

Safety evaluation in the early stage of drug discovery is crucial for the selection of a drug candidate among many compounds. Safety was defined by White [7] as sufficient selectivity for the target receptor so that an adequate dose range exists in which the intended pharmacological action is essentially the only physiological effect of the compound. The ideal system would generate "go/no-go" decision employing only a small amount of the compounds [7]. Pharmaceutical industry is challenged on how to prioritize scale up 


\section{Research costs during product development and their interfaces}

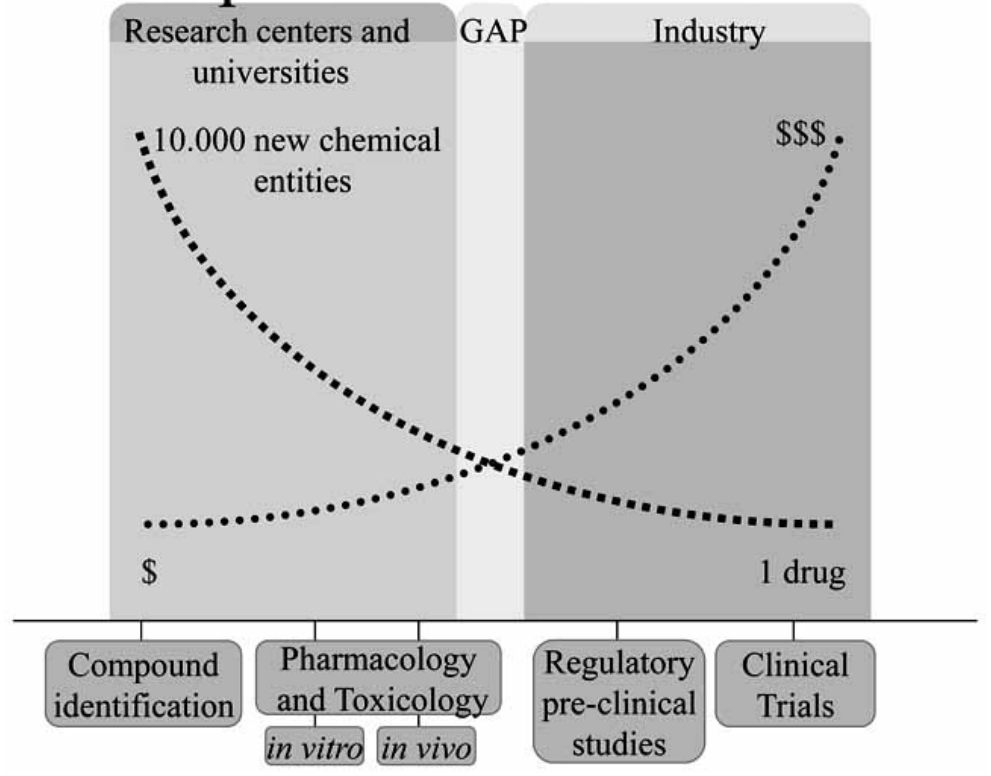

Fig. (1). Public-private-partnerships and their interfaces in drug development process.

of potential leads, considering the quantity (grams) required for secondary testing and safety assessment and the difficulty in obtaining large amounts of compound in early stages [6]. Hence, each step has to be planned to obtain the data required with minimum effort.

In 1997 a report was published pointing out the main reasons for failures in drug development, which were attributed to poor pharmacokinetics $(39 \%)$ and animal toxicity (11\%) [8]. Pharmacokinetic prediction is a hard task once absorption, distribution, metabolism and excretion (ADME) experimental screens are multi-mechanisms and the accumulation of data turns it into a complex work. In long term the advent of a single mechanism ADME assay and biology systems may modify the drug discovery process with its application being prior to target identification [5,9].

Because the majority of discovery projects are focused on the development of orally bioavailable drugs, lead compound selection needs to correlate in vitro data to in vivo absorption. Multiple assays and approaches can be applied for decision making. After the hit identification by pharmacological in vitro studies, lead-like physicochemical properties, cytotoxicity, hepatocyte screening system assays are the next step followed by in vivo pharmacological studies. The synergistic combination of all this information generated is crucial for the selection of lead compounds.

\section{LEAD-LIKE PHYSICOCHEMICAL PROPERTIES}

The investigation of chemical properties can be used to predict in vivo performance of a compound or even to optimize a lead. A successful drug is an intricate balance of bio- logical activity and drug-like properties. Absorption and first-pass metabolism in the liver or gut wall are generally responsible for the amount of compound orally administered found in systemic circulation. Solubility and lipophilicity together determine the extent and rate of absorption and have a direct influence in oral bioavailability, defined as the range of the oral dose that reaches systemic circulation [10].

Over the last years many scientists reported physicochemical properties related to the "drugability" of a compound and that a correlation of partition coefficient $(\log \mathrm{P})$, molecular weight, the number of hydrogen bonding groups and bioavailability of a compound could be established [11, 12].

By computational analyses of the World Drug Index and previous knowledge of chemical properties, researchers from Pfizer proposed that simple chemical rules could be used prospectively to compare and prioritize groups of compounds and different chemical series with greater potential to become orally active compounds. A high percentage of compounds that entered clinical trials, with the desired drug-like properties, was found to have: hydrogen bond donors $\leq 5$; hydrogen bond acceptors $\leq 10$; relative molecular weight $\leq$ 500; and calculated octanol-water partition coefficient (prediction of the ability of a molecule to cross biological membranes) $\leq 5$ [5]. Those rules were called Lipinski's "rule-offive" and became a guide to identify and select small molecules intended to be orally administered.

Although it raised the awareness that drug-like compounds exhibit physicochemical properties that are important for successful drug development, the need to go further Lip- 
inski's rules in recent years brought extended versions with evolution of concepts with the aim to provide quality leads [13]. Lipinski's "rule-of-five" is certainly necessary but not sufficient to create an oral drug-like molecule [14]. Researchers from GlaxoSmithKline, after oral bioavailability measurements in rats for over 1100 drug candidates, suggested that compounds with 10 or fewer rotatable bonds (as a measure of reduced molecular flexibility), and 12 or fewer $\mathrm{H}$-bond donors and acceptors (as a measure of low polar surface area) will have a high probability of good oral bioavailability in the rat [15]. The main limitations of these rules are false positives (compliant compounds are not automatically good drugs) and restricted application (applies only to compounds administered orally and absorbed by passive mechanisms). Besides, there are important exceptions, such as natural products that, over the evolution, might have been optimized by nature to take advantage of active transport or have developed special conformational features that are beneficial for passive transport [13]. Natural products structures present different properties when compared to libraries of synthetic and combinatorial compounds, such as high chemical diversity and biochemical specificity [16].

With emerging valuable tools, it is up to drug development scientists to combine multiple assays and approaches that will permit the selection of lead compounds with physicochemical parameters that will benefit orally bioavailable drugs.

\subsection{Solubility}

The aqueous solubility of a substance is crucial information that has to be readily defined in the very beginning of drug development. Poor solubility and permeability lead to poor bioavailability and lack of in vivo activity [17]. Solubility at a given $\mathrm{pH}$ is influenced by the basic or acidic functional groups of a compound. The solubility of a compound in aqueous media is greater when it is in ionized state [18]. Lipinski estimated the required level of compound solubility to minimize poor absorption. It was found that, for an orally active drug, a compound with medium intestinal permeability and human potency of $1 \mathrm{mg} / \mathrm{kg}$ needs a minimum thermodynamic aqueous solubility of $52 \mu \mathrm{g} / \mathrm{ml}$ (at $\mathrm{pH} 6.5$ or 7.0 ) [5]. The level of solubility needed for oral absorption is related to the potency and permeability, although oral activity can still be achieved with lower solubility when a compound has great potency and is highly permeable. On the other hand, solubility needs to be higher in cases when a compound is not very potent and permeable [17].

\subsection{Permeability}

The two most important determinants of in vivo intestinal absorption are solubility and intestinal permeability [7]. Permeability and solubility are independent and its determinant molecular components are molecular size and hydrogen bonding capacity. An increase in molecular weight and lipophilicity will increase permeability and decrease solubility, whereas the increase hydrogen-bonding capacity and charge will increase solubility and decrease permeability. The orally absorbable compound is a resultant of a balance between the different physicochemical properties. Because the change of one molecular component will affect the other, when a deci- sion needs to be taken, preference should be given to more permeable compounds since it is possible to improve solubility using adequate pharmaceutical technology [17].

During the last few years an increased use of in vitro absorption model, such as Caco-2, a human colon epithelial cancer cell line, was observed in many research fields, such as pharmaceutical sciences for the studies of absorption, permeability and transepithelial transport of drugs [19]. This approach measures rates of compound diffusion down a concentration gradient across cultured Caco- 2 cell monolayers. Apparent permeability coefficients can be calculated by the measurement of the net flux of a compound over this cellular barrier followed by LC/MS/MS (liquid/chromatography/ tandem mass) assay with cells cultured in semi-permeable plastic supports. These coefficients represent rates of permeability for each compound tested [20,21]. This permeability experiment can be automated, allowing rates of a few hundreds of compounds to be performed. Although this system cannot be considered high-throughput screening, it is better than manual cell culture methods [7].

One of the drawbacks of the Caco- 2 cell lines is that, when compared to normal intestinal enterocytes, they present an over-expression of CYP1A1 and a down-regulation of CYP3A4, resulting in differences in metabolic competence [22]. This can be restored by the use of a genetically engineered Caco-2 variant expressing high levels of CYP3A4, allowing assessment of permeability and gut wall metabolism and improving predictability [19].

\subsection{Lipophilicity}

Lipophilicity can be expressed as a partition coefficient between octanol and aqueous phases. When dividing the bioavailability of drugs into absorption and metabolism it is clear that pharmacokinetic parameters are closely related to lipophilicity: high lipophilicity increases metabolic clearance and limits solubility, causing poor absorption [22]. It significantly impacts ADME properties and can be improved by increasing molecular size and decreasing hydrogen-bonding capacity of a compound [17]. The partitioning of a compound is dependent on the $\mathrm{pH}$ of the solution that directly affects its ionization state [18]. They can be estimated very early by simple calculations and guide the choice or design of molecules with acceptable pharmacokinetic profiles.

\section{IN VITRO CELL SYSTEMS}

Cell system models can be designed to scan a wide range of molecules more rapidly and cost effectively than in vivo studies. In a drug discovery process, two initial tests must be applied for predictive toxicology profiling: cytotoxicity and hepatotoxicity. These assays require minimal amounts of compounds, are not time-consuming and, in combination the information generated from these well-established systems, may be a valuable tool for decision-making. Detection and removal of compounds presenting hepatotoxicity and cytotoxicity before getting into the more costly phase should be the goal during discovery phases. Hepatotoxicity can be predicted by a combination of computational and in vitro systems, depending on the availability of time and information needed at a given stage. 


\subsection{Cytotoxicity}

Cytotoxicity is an important parameter in drug development and in vitro determinations are well established in the pharmaceutical and biotechnology industries. It has been used previously for predicting acute toxicity. There are different methodologies for evaluating cell toxicity and assays using 3T3 fibroblast cell line and those based on neutral red uptake are now accepted by authorities from European Union and OECD for regulatory purposes. Biokinetic properties can be predicted by results of in vitro methods and high or medium throughput screening (HTS/MTS) can guide preclinical studies [20]. Regardless of the chosen methodology, a cytotoxicity assay aims to guide the prediction of in vivo toxicity and is a tool for lead compound selection.

A drug candidate should have selective toxicity. Many promising compounds active against protozoans already tested in vitro have no cell cytotoxicity determined. Molecules against intracellular parasites should be able to cross the cell membrane and act specifically on the microorganism. The use of cytotoxicity determination is very well established, and aims to identify hits among the compounds screened in in vitro assays. The purpose of this determination is to ensure that an inhibition in a cell-based assay is not due to inhibited cell viability. Information about adsorption, distribution, metabolism and excretion associated to the cytotoxicity evaluation can reveal an early preclinical profile of the compound [20].

V79, L929, Ehrlich, 3 T3 fibroblasts, HepG2, Vero, Caco2 and murine splenocytes are examples of very common cells used in cytotoxicity determination assays [23-27]. Nowadays, the reduction in the number of animals in research is stimulated by international, and the substitution of freshly isolated cells by cell lines is an option [28]. The readouts of the viability assessment assays vary, and can be based on diverse assay methodologies, such as fluorimetry, luminescence, scintillation, colorimetry, cell staining/dye exclusion, among others.

There are diverse methodologies based on fluorimetry for cytotoxicity assessment. In general they are based on the measurement of signaling molecules liberated by non viable cell or on staining of nucleic acids, such as calcein/ethidium homodimer method, which allows to evaluate cell viability/cytotoxicity [29]; the glucose-6-phosphate released by damaged and dying cells, which can be detected by an enzymatic process including the reduction of resazurin into red-fluorescent resorufin [29]; and propidium iodide (PI), a molecule able to bind to DNA between the bases, which only penetrates in dead cells [30].

PI is a toxic molecule, which can induce mutation and should be handled with care. This characteristic does not invalidate the method because, if handled with care, PI is absolutely secure and does not generate radioactive residues. Cytotoxicity analysis using PI associated with flow cytometry is a sensitive and precise method and can be employed in analysis of normal or neoplastic murine or human cells, since PI to nucleic acid in not viable cells. The existence of automated high-speed processing for this method should be considered, which facilitates its use, as reported by Nunez [31]. Assays based in fluorimetry analyzed by flow cytometry permit high-content screening reflecting some of recent efforts to advance its automation and more efficient application to the drug discovery process [32]. When a compound has a natural color the evaluation of cytotoxicity by colorimetric assays may be difficult due to its influence on the spectrophotometric analysis. In the PI viability assessment, however, there is no interference of the compound color, since the technique is based on emitted fluorescence resultant of the PI binding to the nucleic acid.

Regarding methods based on luminescence, the ATP/ luciferase can be cited as an example of method for detection of viable cells, since the luminescence production by luciferase requires ATP [29]. Cell lines transfected with the luciferase gene can be used in this luminescence-based cell viability assays.

Among methods based on scintillation count, ${ }^{3} \mathrm{H}$-thymidine uptake is largely used. Thymidine is a nucleoside that can be incorporated by viable cells in their nucleic acids metabolism in non-stimulated and stimulated cell cultures. When cell proliferation is stimulated, it induces the production of nucleic acid, which requires nucleosides. When ${ }^{3} \mathrm{H}-$ thymidine is added to the culture medium, the labeled nucleotide competes with endogenously available thymidine for incorporation into newly synthesized nucleic acid [33, 34]. Other viable eukaryotic cells can do the same, by the described mechanism, including neoplastic lineages. In nonstimulated cells there is a basal t-RNA metabolism also that requires thymidine $[33,34]$. Thus, splenocytes can incorporate thymidine in a lowest proportion, but enough to quantify if compared with cultures with non-viable cells. The incorporation of ${ }^{3} \mathrm{H}$-thymidine can be measured in a scintillation counter, expressing proportional scintillation value to the cell viability. Quantification can be accessed by detecting radiolabeled nucleic acid, using glass-fiber filter mats or plates, which trap genomic molecules along with some cell fragments but spare oligonucleotides or single bases [33]. The method based on ${ }^{3} \mathrm{H}$-thymidine uptake is very practical because it is possible to perform the assay in 96-well plates, allowing the screening of a large quantity of compounds/ plate. In addition to the advantages cited above, this method also eliminates the color interference in when a colorful compound is being analyzed. This method has a disadvantage, which is the generation of radioactive solid and liquid waste. Manipulation should be carried out by trained personnel and care should be adopted aiming to protect the laboratory area, other researchers and the environment.

Common colorimetric assays are based in cell metabolism of one substrate producing a colorful product, like formazan, using lactate dehydrogenase (LDH) or MTT methods. LDH, an enzyme released from damaged cells, oxidizes lactate to pyruvate which reacts with tetrazolium salt producing formazan. MTT (4,5-dimethylthiazol-2-yl)2,5-diphenyl tetrazolium bromide) is a tetrazolium salt that active mitochondria can cleave [35]. This is an assay useful, secure, simple and fast to measure cell viability in culture. The MTT cleavage is used for cell viability evaluation or cell proliferation [36], and can be used to determine small alterations in the cell redox status and changes in mitochondrial activity. Since the introduction of MTT for cell viability evaluation in 1983, many other tetrazolium salts have been used, such as 
XTT, MTS, and WST [35]. Among the many methods for cell viability that evaluate the cell oxidative capacity, the MTT method has been the most used because of its sensitivity and reliability [37]. Although the cell that does not metabolize MTT may only be presenting some deficiency in the oxidative metabolism, without being necessarily dead, the quantity of formazan produced is usually considered as directly proportional to the total number of active mitochondria by cell in culture [38]. MTT method has some advantages in comparison to other methods. It does not produce radioactive residues, is not an expensive method and adherent and non-adherent cells can be used in high-throughput screening processes. Disadvantages of the method include the difficulty of analysis of heterogeneous cell populations due to differences in the cell metabolism individual capacity and interference of colorful compounds in absorbance values and, therefore, in the cytotoxicity results.

Cell viability assessment by trypan blue (TB) exclusion is a very useful, practice and cheap method. The cytotoxicity is determined counting cells manually, using TB solution, which is a dye used to evaluate cellular death. Viable cells, when observed through optical microscope, stay uncolored, while dead cells appear stained in blue. Cell membrane of non-viable cells allows the absorption of TB. Some disadvantages of this method can be listed, such as the quantification is made by manual counts, requiring longer time and allowing procedure errors, or the need to remove adherent cells (e.g. using trypsin) for counting. Considering this, the use of this technique characterizes a low-throughput screening process.

\subsection{Selectivity Index}

The selectivity index (SI) is a parameter that can be used to determine drug specificity for any activity. The SI is calculated dividing the $\mathrm{LC}_{50}$ by $\mathrm{IC}_{50}$ values. This index can be related to any biological activity like antiprotozoal, immunomodulatory or antineoplastic activity. The SI is considered significant when its value is higher than three [24] and the more elevated is the drug selectivity index, the higher is the drug more selectivity. One example of a highly selective drug is nifurtimox (an anti-Trypanosoma cruzi agent), which presents an SI equal to 154 [39]. Another example is chloroquine, an antimalarial agent showing a SI equal to 200 for a chloroquine-sensitive Plasmodium strain while the same drug shows a low SI (20) for a chloroquine-resistant strain, as reported by Desoubzdanne [40].

\subsection{Hepatocyte Screening System}

Drug-induced liver injury is the most frequent reason for the withdrawal of an approved drug from the market and inclusion of black box warning [41]. Between 1979 and 1999,45 previously approved drugs received one or more black box warning (10 due to hepatotoxicity) and 16 were withdrawal from the market because of safety reasons ( 5 due to hepatotoxicity) [42]. Currently the investigation of drug metabolism and toxicity is done much earlier in the discovery process. The central role of hepatocytes in the metabolism of xenobiotics with the generation of intermediate or final metabolites with potential toxic effect for the organism places the liver into a central context.
During the discovery lead optimization process a hepatocyte screening system should provide data on (Fig. (2)):

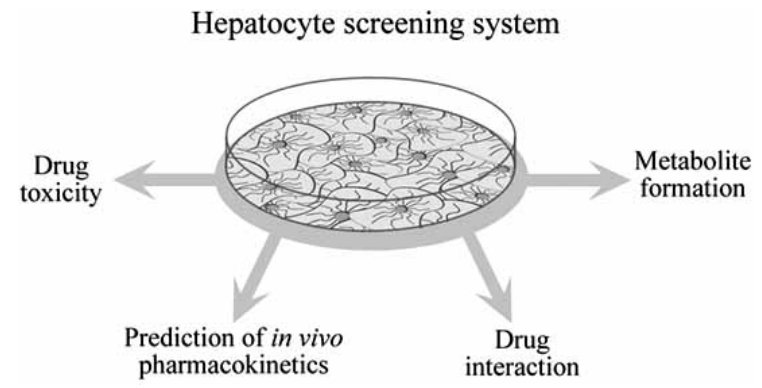

Fig. (2). In vitro methods performed with hepatocytes, microssomes and CYP cultures for prediction of drug toxicity, metabolite formation, drug-drug interaction and pharmacokinetics in vivo.

- Drug toxicity - provides the opportunity to study in detail mechanisms of hepatotoxicity in comparison with in vivo conditions.

- Drug-drug interaction - can be predicted by the detection of substrates inhibitors or inducers of the enzyme involved in the metabolic pathway of a compound.

- Metabolite formation - the formation of reactive metabolites can be investigated by the use of primary hepatocytes.

- Prediction of in vivo pharmacokinetics - preliminary prediction of the in vivo behavior of a compound with potential to become a drug.

Drug metabolism is a crucial determinant of drug clearance and interindividual pharmacokinetic differences, leading to efficacy or failure of a given drug candidate during clinical test [43]. Gene polymorphisms can be the reason for interindividual differences concerning drug response once there is no standard profile for CYP expression in man $[44,45]$.

Among the whole universe of CYP isoforms found in human liver (Fig. (3)), attention must be paid to the ones known to have the highest impact on drug metabolism. CYP3A4 is the most abundant enzyme in the liver and is involved in the metabolism of over 50\% of drugs [46]. Because of its high content in the liver and history of well conserved genes this enzyme represents a good choice for selecting a high affinity lead candidate in a drug screening program. However, there are other factors, such as sex, age, use of ethanol and other drugs underlying diseases that can modulate gene expression, resulting in susceptibility for adverse reaction. Those events are often unpredictable and will be detected in late stages during clinical trials of drug development [47].

On the other hand, a drug design avoiding compounds that present high affinity substrates for CYP2D6 is desired. Although present in low amounts in human liver (Fig. (3)), this enzyme metabolizes $20-25 \%$ of drugs used clinically and its high polymorphism represents huge differences in the clinical response that goes from reduced pharmacologic effect (for the ultra-rapid metabolisers) to toxicity (for the poor 
metabolisers) [45]. In addition, clinically relevant polymorphisms have been identified for de CYP 2C9 and 2C19 and this has a profound effect in drug efficacy and toxicity $[48,49]$.

\section{Content of CYP isoforms in human liver}

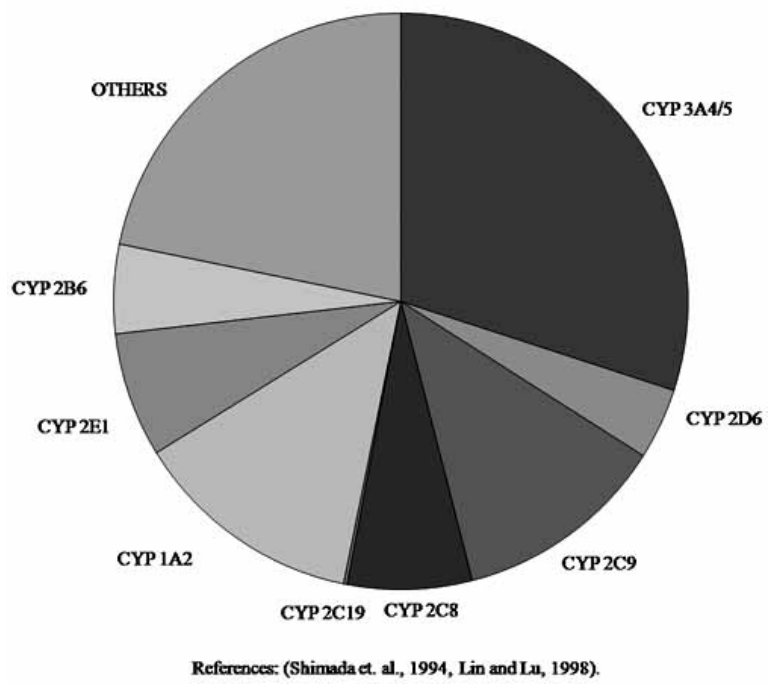

Fig. (3). The estimated content of CYP isoforms in human liver according to key references.

\subsubsection{Drug Toxicity}

It is hard to detect hepatotoxicity occurring at low incidence with the number of animals used in toxicology studies. Considering that it is time consuming and costly to perform in vivo studies with massive number of drugs, robust in vitro screening assays are being developed for prediction of human hepatotoxicity caused by drugs [49-51].

Drug toxicity is the most common cause of acute liver failure. It is a serious health problem that impacts society and represents a major impediment in drug development [52]. Hepatotoxicity can be caused by the direct action of a drug or, more often, a reactive metabolite of a drug against hepatocytes or by drug-induced immune-mediated liver injury [53].

One of the predominant forms of drug-induced liver injury is acute hepatitis, defined as a marked increase in aminotransferases coinciding with hepatocellular necrosis [53]. Hepatic necrosis can be detected in nearly $50 \%$ of cases reported [54]. Apoptosis can also be detected in hepatocyte models and is an important parameter used in the toxicity analysis of xenobiotics [55].

A cytotoxicity assay specifically developed to predict clinical acute hepatotoxicity (acute liver failure) based on immortalized cell lines representing an exaggerated case of metabolizing enzyme has been used early in discover process. The CYP450 enzyme isoforms and the 3A4, 2C9, 2C19 and 2D6 cell lines are the basis of an assay that determines
$\mathrm{IC}_{50}$ values and can be used to evaluate the potential for hepatotoxicity across candidate compounds for lead optimization ranking [49].

\subsubsection{Prediction of In Vivo Pharmacokinetics}

Metabolism in animals differs from that in humans, and thus the prediction of human pharmacokinetics from animal data might not be straightforward, being crucial to determine previously the specific CYP involved in the drug metabolism in order to select an appropriate in vivo system. As reviewed by Zuber [46], for CYP1A mediated pathways, with the exception of the dog, all the experimental models commonly used are appropriate, whereas for drugs metabolized by CYP2D the dog seems to be the most suitable. The CYP3A seems to be well modeled in pigs, whereas a good alternative for CYP2C may be a system based on monkeys (Maccacus rhesus) [46].

A major challenge in this phase is to find a model that provides high throughput data in order to screen a large number of compounds. As reviewed by $\mathrm{Yu}$ and Adedoyin [56], integration of experimental and computational technologies can be used to characterize ADME profile of a compound. The assay applied will depend on the stage of the process. An early discovery stage demands the evaluation of hundreds or thousands of compounds quickly and does not require the details and depth of data necessary in the next stages. In silico ADME models may soon serve as a filter for a large number of compounds before in vitro ADME toxicological tests [57]. Each therapeutic class has its own pharmacokinetics requirements. Therefore, the range of ADME must be very comprehensively and carefully studied in each phase of the program [58].

\subsubsection{Drug Interaction}

Reduced pharmacological effect or increased toxicity of a drug (by production of reactive intermediates) may be observed when the drug tested is co-administrated with another drug that causes increased activity of one or more enzymes [44,59]. A competitive or noncompetitive inhibition of a CYP 450 enzyme responsible for the metabolism of a coadministered drug, leading to its accumulation, can also be observed. In case the drug has inherent toxicity, adverse effects may be observed [49]. This phenomenon is called drugdrug interactions and has serious clinical consequences.

It is important to identify the metabolic pathways of candidate compounds by conducting in vitro evaluation of changes in CYP 450 enzyme mRNA level and monitoring enzyme activity $[49,60]$. The identification of drug metabolizing enzymes helps to predict the drug-drug interaction that may occur in man [58]. Most of the drug clearance is mediated by five CYP isoforms, 3A4, 2D6, 2C9, 1A2, and 2C19 [44], and therefore these should be the ones tested.

Inhibition of cytochrome $\mathrm{P} 450$ is a principal mechanism for metabolism-based drug-drug interactions. The development of high-throughput CYP-mediated cocktail assay with quantification by liquid chromatography/tandem mass spectrometry (LC/MS-MS) has a potential demonstrated using drug library compounds, which showed that this assay has application in early-stage drug discovery $[59,61]$. 
Drug-drug interaction is not a direct measurement of in vitro CYP450 inhibition assay. It occurs when two coadministered drugs compete for the same CYP enzyme and when the metabolic reaction catalyzed by this enzyme is the major elimination pathway [62]. Hence, all the data generated must be carefully analyzed.

\subsubsection{Metabolite Formation}

The rate of metabolism of a drug can be determined using in vitro cell systems by the measurement of the drug disappearance over time, as well as metabolite formation [44]. Active metabolites of several marketed drugs have been developed as drugs with better efficacy and ADME profile [59]. Characterization of metabolites is critical for optimizing lead compounds with improved metabolic stability and toxicological profile $[44,62]$. Otherwise, compounds that form toxic metabolites that could cause adverse effects can be eliminated [63].

Metabolites can be generated with the use of animal and human recombinant expressed enzymes, human liver and hepatocytes and pre-clinical animal models [58,63]. The metabolite structure elucidation can be determined by the combined use of accurate mass liquid chromatography/tandem mass spectrometry (LC/MS/MS). This methodology integrates the parent disappearance, metabolite identification, and the formation of the metabolites along the time course using a single rapid LC/MS/MS analysis [64,65].

Throughput studies for drug metabolite identification have increased significantly in the last years. Although it is still too labor-intensive in the drug discovery setting, recent publications anticipate that the combination of LC/MS/MS and LC/NMR will be crucial for structure metabolite elucidation and feasible to apply in early drug discovery [66].

\section{CONCLUDING REMARKS}

ADME tests begin with the identification of a hit and continue during lead optimization, selection and development stages [58]. Unsuitable ADME is, together with animal toxicity, responsible for half of all failures in drug development [57]. At the lead compound selection phase, there is a need for high throughput methods to screen many hits. Although there is no need to go depth into the information, early data related to hepatotoxicity, ADME profile and cytotoxicity are crucial. All of these parameters are interrelated and need to be considered in parallel. There is a need for new technologies to be explored as a way of reducing drug attrition rates and either in academia or industry these key questions (toxicity and ADME profile) must be answered. It is important to point out that the methods discussed here are predictions of in vivo behavior of a compound. The safety of new compounds is multimechanism determination dependent on each individual's response, and therefore it cannot be known with certainty until a drug has been on the market for many years.

\section{ACKNOWLEDGEMENTS}

This work was supported by grants from CNPq, FINEP, MCT (IMSEAR, Institutos do Milênio), and FAPESB. MBPS and RRS are senior researchers for CNPq (Brazil).
ABBREVIATIONS

\begin{tabular}{|c|c|c|}
\hline ADME & $=$ & $\begin{array}{l}\text { Absorption, distribution, metabolism and } \\
\text { excretion }\end{array}$ \\
\hline ATP & $=$ & Adenosine triphosphate \\
\hline CYP & $=$ & Cytocrome P450 \\
\hline $\mathrm{DL}_{50}$ & $=$ & Lethal dose for $50 \%$ \\
\hline DMSO & $=$ & Dimethyl sulfoxide \\
\hline DNA & $=$ & Deoxyribonucleic acid \\
\hline G6P & $=$ & Glucose-6-phosphate \\
\hline $\mathrm{IC}_{50}$ & $=$ & Inhibitory concentration for $50 \%$ \\
\hline LC/MS/MS & $=$ & Liquid/chromatography/tandem mass \\
\hline $\mathrm{LC}_{50}$ & $=$ & Lethal concentration for $50 \%$ \\
\hline MTT & $=$ & $\begin{array}{l}\text { 4,5-dimethylthiazol-2-yl)2,5-diphenyl } \\
\text { tetrazolium bromide }\end{array}$ \\
\hline NADPH & $=$ & $\begin{array}{l}\text { Reduced nicotinamide adenine dinucleo- } \\
\text { tide phosphate }\end{array}$ \\
\hline OECD & $=$ & $\begin{array}{l}\text { Organisation for economic co-operation } \\
\text { and development }\end{array}$ \\
\hline PBS & $=$ & Phosfate buffered saline \\
\hline $\mathrm{pH}$ & $=$ & Potential hydrogenionic \\
\hline PI & $=$ & Propidium iodide \\
\hline RD & $=$ & Research and development \\
\hline RNA & $=$ & Ribonucleic acid \\
\hline SI & $=$ & Selectivity index \\
\hline TB & $=$ & Trypan blue \\
\hline t-RNA & $=$ & Transporter ribonucleic acid \\
\hline WST or XTT & $=$ & $\begin{array}{l}\text { 2,3-bis[2-methoxy-4-nitro-5- } \\
\text { sulphophenyl]-2H-tetrazolium-5 } \\
\text { caroxanilide }\end{array}$ \\
\hline
\end{tabular}

\section{REFERENCES}

[1] Nwaka, S. and Hudson, A. (2006) Nat. Rev. Drug Discov., 5, $941-$ 955.

[2] Croft, S.L. (2005) Trans. R Soc. Trop. Med. Hyg., 99(1), 9-14.

[3] Bathurst, I. and Hentschel, C. (2006) Trends Parasitol., 22(7), 301307.

[4] Dickson, M. and Gagnon, J.P. (2004) Nat. Rev. Drug. Discov., 3(5), 417-429.

[5] Lipinski, C.A.; Lombardo, F.; Dominy, B.W.; and Feeney, P.J. (1997) Adv. Drug Del. Rev., 23, 3-25.

[6] Bugelski, P.J.; Atif, U.; Molton, S.; Toeg, I.; Lord, P.G. and Morgan, D.G. (2000) Pharm. Res., 17(10), 1265-1272.

[7] White, R.E. (2000) Ann. Rev. Pharmacol. Toxicol., 40,133-157.

[8] Kennedy, T. (1997) Drug Discov. Today, 2, 436-444.

[9] Butcher, E.C. (2005) Nat. Rev. Drug Discov., 4(6), 461-467.

[10] Walters, W.P. and Ajay, M.M.A. (1999) Curr. Opin. Chem. Biol., 3(4), 384-387.

[11] Navia, M.A. and Chaturvedi, P.R. (1996) Drug Discov. Today, 1(5), 179-189.

[12] Chan, O.H. and Stewart, B.H. (1996) Drug Discov. Today, 1, 461473.

[13] Keller, T.H.; Pichota, A. and Yin, Z. (2006) Curr. Opin. Chem. Biol., 10(4), 357-361.

[14] Hann, M.M. and Oprea, T.I. (2004) Curr. Opin. Chem. Biol., 8(3), 255-263. 
[15] Veber, D.F.; Johnson, S.R.; Cheng, H.Y.; Smith, B.R.; Ward, K.W. and Kopple, K.D. (2002) J. Med. Chem., 45(12), 2615-2623.

[16] Clardy, J. and Walsh, C. (2004) Nature, 432, 829-837.

[17] Li, Y.; Shin, Y.G.; Yu, C.; Kosmeder, J.W.; Hirschelman, W.H.; Pezzuto, J.M.; and van Breemen, R.B. (2003) Comb. Chem. High Throughput Screen., 6(8), 757-767.

[18] Chen, I.J.; Taneja, R.; Yin, D.; Seo, P.R.; Young, D.; MacKerell, A.D. and Jr, Polli, J.E. (2006) Mol. Pharm., 3, 745-755.

[19] Artursson P.; Palm K. and Luthman K. (2001) Adv. Drug Deliv. Rev., 46(1-3), 27-43.

[20] Walum, E.; Hedander, J. and Garberg, P. (2005) Toxicol. Appl. Pharmacol., 207(2), 393-397.

[21] Van Breemen, R.B. and Li, Y. (2005) Expert Opin. Drug Metab. Toxicol., 1(2), 175-185.

[22] Bertrand, M.; Jackson, P. and Walther, B. (2000) Eur. J. Pharm. Sci., 11(2), 61-72.

[23] Melo, P.Sl; Durán, N. and Haun, M. (2000) Toxicol. Lett., 116, 237-242.

[24] Bézivin, C.; Tomasi, F.; Lohézic-Le, F. and Boustie, J. (2003) Phytomedicine, 10, 499-503.

[25] Prayoung, P.; Barusrux, S. and Weerapreeyakul, N. (2008) Fitoterapia, 79, 598-601.

[26] Wada, K.I.; Hamaguchi, Y.; Furukawa, K. and Taniguchi, A. (2008) Biotechnology and Bioengineering - Published Online: 21 Oct 2008.

[27] Costa, J.F.O.; Kiperstok, A.C.; David, J.P.L.; David, J.M.; Giulietti, A.M.; Queiroz, L.P.; Santos, R.R. and Soares, M.B.P. Fitoterapia, 78, 510-514.

[28] Gannon, F. (2007) EMBO Rep., 8(6), 519-520.

[29] The handbook - 10th edition - Invitrogen. On-line version, accessed in 12/04/2008 at www.invitrogen.com.

[30] Shapiro, H.-M. Practical flow cytometry, Wiley-Liss: Wiley-Liss, 2003.

[31] Nunez, R. (2001) Curr. Issues Mol. Biol., 3(2), 39-45.

[32] Edwards, B.S.; Oprea, T.; Prossnitz, E.R. and Sklar, L.A. (2004) Curr. Opin. Chem. Biol., 8, 392-398.

[33] Kaufmann, S. H. E.; Kabelitz, D. Immunology of infection, Academic Press: San Diego, 1998.

[34] Lehninger, A.L.; Nelson, D.L.; Cox, M.M. Principles of biochemistry, Ed. Sarvier: New York, 2007.

[35] Nath, B.B.; Babrekar, A.A. and Parthasarathy, B. (2005) Cytotechnology, 49, 59-65.

[36] Ferrari, M.; Fornasiero, M.C. and Isetta, A.M. (1992) J. Immunol. Methods, 153, 265-266

[37] Traore, H.N. and Meyer, D. (2001) Methods Cell Sci., 23, 175-184.

[38] Pabbruwe, M.B.; Stewart, K. and Chaudhuri, J.B. (2005) Biotechnol. Lett., 27, 1451-1455.

[39] Aponte, J.C.; Verástegui, M.; Málaga, E.; Zimic, M.; Quiliano, M.; Vaisberg, A.J.; Gilman, R.H. and Hammond, G.B. (2008) J. Med. Chem., 51, 6230-6234.

[40] Desoubzdanne, D.; Marcourt, R.; Raux, R.; Chevalley, S.; Dorin, D.; Doerig, D.; Valentin, A.; Ausseil, F. and Debitus, D. (2008) J. Nat. Prod., 71(7), 1189-1192.
[41] Park, K.; Williams, D.P.; Naisbitt, D.J.; Kitteringham, N.R. and Pirmohamed, M. (2005) Toxicol. Appl. Pharmacol., 207(2), 425434.

[42] Lasser, K.E.; Allen, P.D.; Woolhandler, S.J.; Himmelstein, D.U.; Wolfe, S.M. and Bor, D.H. (2002) JAMA, 287, 2215-2220.

[43] Gómez-Lechón, M.J.; Castell, J.V. and Donato, M.T. (2007) Chem. Biol. Interact., 168(1), 30-50.

[44] Hewitt, N.J.; Lechón, M.J.; Houston, J.B.; Hallifax, D.; Brown, H.S.; Maurel, P.; Kenna, J.G.; Gustavsson, L.; (2007) Drug Metab. Rev., 39(1), 159-234.

[45] Ingelman-Sundberg, M. (2005) Pharmacogenomics J., 5(1), 6-13.

[46] Zuber, R.; Anzenbacherová, E. and Anzenbacher, P. (2002) J. Cell Mol. Med., 6(2), 189-198.

[47] Kaplowitz, N. (2004) Clin. Infect. Dis., 38(2), 44-48.

[48] Zhou, S.F.; Di, Y.M.; Chan, E.; Du, Y.M.; Chow, V.D.; Xue, C.C.; Lai, X.; Wang, J.C.; Li, C.G.; (2008) Curr. Drug Metab., 9(8), 738784.

[49] Dambach, D.M.; Andrews, B.A. and Moulin, F. (2005) Toxicol. Pathol., 33(1), 17-26.

[50] Gebhardt, R.; Hengstler, J.G.; Müller, D.; Glöckner, R.; Buenning, P.; Laube, B.; Schmelzer, E.; Ullrich, M.; Utesch, D. (2003) Drug Metab. Rev., 35(2-3), 145-213.

[51] Lahoz, A.; Donato, M.T.; Montero, S.; Castell, J.V. and GómezLechón, M.J. (2008) Rapid Commun. Mass Spectrom., 22(2), 240244.

[52] Hussaini, S.H. and Farrington, E.A. (2007) Expert Opin. Drug Saf., 6, 673-684.

[53] Holt, M.P. and Ju, C. (2006) AAPS J., 8(1), 48-54.

[54] Guillouzo, A.; Morel, F.; Langouët, S.; Maheo, K. and Rissel, M. (1997) J. Hepatol., 26(2), 73-80.

[55] Gómez-Lechón, M.J.; O'Connor, J.E.; Lahoz, A.; Castell, J.V. and Donato, M.T. (2008) Curr. Med. Chem., 15(20), 2071-2085.

[56] Yu, H. and Adedoyin, A. (2003) Drug Discov. Today, 8(18), 852861.

[57] Van de Waterbeemd, H. and Gifford E. (2003) Nat. Rev. Drug Discov., 2(3), 192-204.

[58] Singh, S.S. (2006) Curr. Drug Metab., 7(2), 165-182.

[59] Zientek, M.; Miller, H.; Smith, D.; Dunklee, M.B.; Heinle, L.; Thurston, A.; Lee, C.; Hyland, R.; Fahmi, O. and Burdette, D. (2008) J. Pharmacol. Toxicol. Methods, 58(3), 206-214.

[60] Riley, R.J. and Kenna, J.G. (2004) Curr. Opin. Drug Discov. Devel., 7(1),86-99.

[61] Lin, T.; Pan, K.; Mordenti, J. and Pan, L. (2007) J. Pharm. Sci., 96(9), 2485-2493.

[62] Yan, Z. and Caldwell, G.W. (2001) Curr. Top. Med. Chem., 1(5), 403-425.

[63] Baranczewski P.; Stańczak, A.; Kautiainen, A.; Sandin, P. and Edlund P.O. (2006) Pharmacol. Rep., 58(3), 341-352.

[64] Gao, H.; Materne, O.L.; Howe, D.L.; and Brummel, C.L. (2007) Rapid Commun Mass Spectrom., 21(22), 3683-3693.

[65] Tiller, P.R.; Yu, S.; Castro-Perez, J.; Fillgrove, K.L. and Baillie, T.A. (2008) Rapid Commun. Mass Spectrom., 22(7), 1053-1061.

[66] Prakash, C.; Shaffer, C.L. and Nedderman, A. (2007) Mass Spectrom. Rev., 26(3), 340-369. 